\title{
Software for design and analysis of multi-pass absorption cells
}

\author{
A. Belina Brzozowski, M. Winkowski, T. Stacewicz \\ Institute of Experimental Physics, Faculty of Physics, University of Warsaw, \\ 5 Pasteura Street, 02-093 Warsaw, Poland tadeusz.stacewicz@fuw.edu.pl
}

Received: 09.09.2020

\begin{abstract}
We present interactive calculative approach and relevant software for designing multi-pass optical cells. Our technique performs the analysis of these cells for any sets of concave spherical mirrors and finds the optimal parameters for both laser cavity and optical system that controls laser beam.
\end{abstract}

Keywords: laser spectroscopy, trace gas detection, multi-pass cells.

UDC: 535.343 .4

\section{Motivation and significance}

Sensitive and fast gas detectors are used in many branches such as industry [1], environmental studies [2] and agriculture. The examples are monitoring of toxic gases [3], detection of explosives [4] and medical diagnostics (e.g., breath analysis [5, 6]). This implies a great need in cheap, highly sensitive, easy-to-use and portable measuring equipment. Laser absorption spectroscopy often provides the opportunity for constructing selective sensors of specific gaseous species even under conditions of ultra-low gas concentrations. The samples under test can be measured within response times of the order of several seconds. Among various approaches used in the field, multipass absorption spectroscopy represents a well recognized technique.

Multi-pass method is an extension of single-pass spectroscopy [7]. In the both techniques, the concentration $N$ of absorber is determined basing on the Lambert-Bourger-Beer law. Light from a source with the intensity $I_{0}(\lambda)$ (with $\lambda$ denoting the light wavelength) is quenched when passing through a sample. After measuring the radiation intensity at the sample output, $I(\lambda)$, one can use the relation

$$
N=\frac{1}{\sigma(\lambda) L} \ln \left[\frac{I_{0}(\lambda)}{I(\lambda)}\right],
$$

where $\sigma(\lambda)$ denotes the absorption cross section and $L$ the path length of light in the sample. In order to achieve high sensitivity, one has to tune the source wavelength to the spectral region where the cross section for the compound under interest is the highest. Moreover, the selectivity of detection requires choosing the wavelength $\lambda$ at which the absorption of the compound dominates over the light quenching by the other species.

Note that the single-pass spectroscopy is usually not sensitive enough for sensing traces of gases, since the difference between the $I_{0}(\lambda)$ and $I(\lambda)$ values is too low. The sensitivity of the multi-pass absorption spectroscopy is much higher because it exploits greatly lengthened light path in the sample. A typical multi-pass absorption cell is equipped with two (or more) mirrors which are precisely located with respect to each other. A light beam is introduced into a cell through a hole in one of the mirrors and then reflects multiply among them (see Fig. 1). As a result, a beam 
passes repeatedly through the sample. The effective light paths of tens of metres or even more can be achieved in this way. A compact size and a small volume are additional advantages of the multi-pass approach.

a)

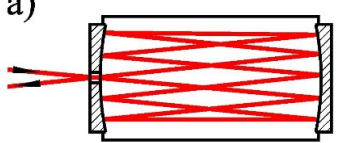

b)

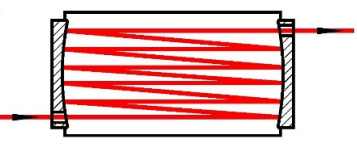

Fig. 1. Examples of multi-pass cells.

The idea of multi-pass spectroscopy has been suggested by J. U. White in 1942 [8] and D. Herriot et al. [9]. The contemporary designs are often based on those topologies. The cells with the optical paths as long as about $100 \mathrm{~m}$ combined with small volumes have been reported in the literature [3, 8]. J. Altmann et al. [10] have described two-mirror multi-pass absorption cells with the effective absorption lengths reaching $150 \mathrm{~m}$. Finally, white-type open cells with the paths exceeding $1 \mathrm{~km}$ have also been suggested [4].

In addition to ultra-sensitive absorption measurements, the constructions mentioned above are applied for the other purposes. D. Herriott et al. [9] have developed a theory of the devices used for multi-pass interferometry and folded optical delay lines [11]. They have also considered aspherical cells, by either applying astigmatic mirrors or using a small perturbing mirror. The both approaches increase the number of multiple passes through a cell, with clear separation of input and output spot. This yields in the optical constructions with extremely large path lengths. The theory enables one to determine basic principles of the cells. First of all, the system must be stable, so that the focal length $f$ of a mirror and the separation distance $d$ between mirrors have to meet the following requirements [9]:

$$
0<\frac{d}{f}<4
$$

In practice, only the cells with $d<2 f$ are used, since some laser spots on the mirrors could become too large in the case of longer constructions, thus leading to spot overlapping. As a consequence, unexpected light interferences could occur. That would cause a so-called 'optical noise', a non-uniform transmission spectrum of the cell which complicates the analysis of weak absorption spectra.

\section{Formulation of problem}

Adjustment of a multi-pass cell, its performance and matching of the directions of input light beams are complex and time-consuming issues, since consistent coordination of many degrees of freedom is necessary. Selection of the mirrors for multi-pass experiments involves their radii $(M R)$, the radii of curvature $\left(R_{1}\right.$ and $\left.R_{2}\right)$, the radii of holes $\left(r_{H 1}\right.$ and $\left.r_{H 2}\right)$ and their distances from the mirror centres ( $R_{H 1}$ and $R_{H 2}$ - see Fig. 2). Moreover, many other parameters should be chosen and coordinated properly in order to achieve a long-path device. These are the mirror separation distance (sep) and the tilting angles $\left(\theta_{X 1}, \theta_{Y 1}, \theta_{X 2}, \theta_{Y 2}\right)$ of the mirrors with respect to the cell axis. The rotation angles of the mirrors (i.e., the hole positions $\varphi_{1}$ and $\varphi_{2}$ ) must also be taken into account. The adjustment of external optics is important, too. This requires a proper choice of the input-laser beam angles $\left(\alpha_{X}, \alpha_{Y}\right)$ with respect to the cell axis. 

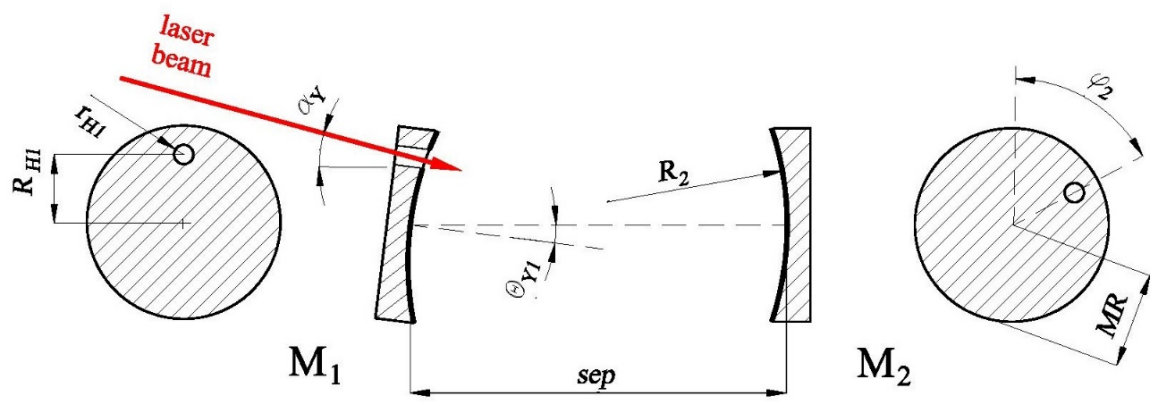

Fig. 2. Vertical sections of optical resonator, with explanation of some related parameters (see the text).

As a result, the path length of light represents a complicated multi-peak function of all the parameters mentioned above. The example is presented in Fig. 3, where the maximal path is shown depending on the sep value. Here the calculations have been performed basing on the approach presented in Section 3 below. The mirror separations have been taken with the step of $1 \mathrm{~cm}$. Here the scheme of Fig. 1a is analyzed. The modelling is done for the mirrors with the radius $M R=1$ " and the radii of curvature $R_{1}=R_{2}=200 \mathrm{~cm}$. It is assumed that the input/output hole with the radius $r_{h 1}=0.2 \mathrm{~cm}$ is located in the centre of the mirror $\mathrm{M}_{1}\left(R_{H 1}=0\right)$, while the mirror $\mathrm{M}_{2}$ is entire (i.e., it has no hole). The optimal values $\theta_{X 1}, \theta_{Y 1}, \theta_{X 2}, \theta_{Y 2}$ and the $\varphi_{X}$ and $\varphi_{Y}$ angles have been found with the Monte-Carlo technique.

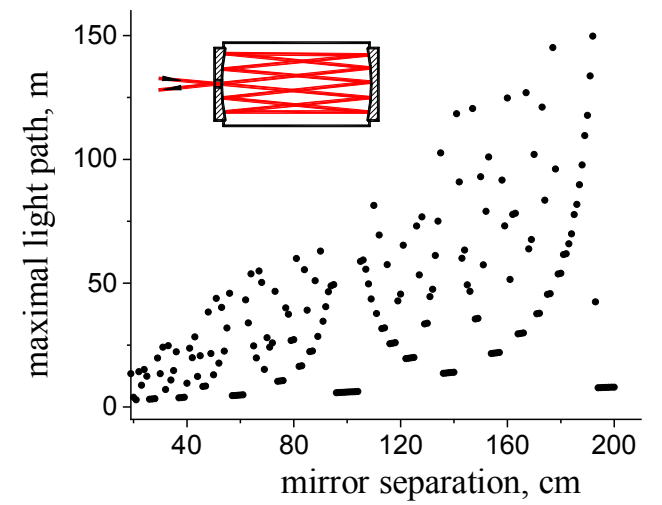

Fig. 3. Maximal light path reached in a multi-pass cell calculated as a function of distance between mirrors.

The results of these calculations demonstrate that, for given mirrors, a poor selection of the distance during the cell design can finally cause insufficient number of multiple beam passes and so a short overall optical path. Notice that this cannot be compensated by selection of beam and mirror angles.

Although some authors have tried to describe the design of their multi-pass cells [12], usually there is no information enough for reproducing exactly their experiments in other laboratories. The data provided is typically limited to the mirror type, the radius of curvature and the optimized optical path. Moreover, a great number of the parameters make the analytical description of the problem hardly feasible. It would be much more beneficial to use the appropriate computer simulations.

In this work we present a method and software which aid in designing custom multi-pass cells. In particular, we suggest an open-source, free-of-charge and user-friendly tool for simulating 
and optimizing the optical construction of the cells. The software enables one to perform interactive analysis of the multi-pass cells for any sets of spherical concave mirrors and find easily the optimal parameters for the laser cavity and the optical system which supplies the laser beam.

\section{Software description}

Our solution is based on geometrical optics and analytic geometry. First, the equations for each reflecting sphere and a straight line representing input light beam are written. Then the coordinates of intersection point of the beam and the opposite mirror surface are calculated. The next step consists in finding the equation of reflected ray. Then sequential reflection points and reflectedbeam directions are determined (see also Appendix 1).

Our approach approximates each ray as a straight line. Nonetheless, the studies that involve the beams of certain 'thicknesses' and divergences might also be conducted. In the latter case, we analyze the propagation of four additional lines that generate a cone representing the beam. This allows searching for consecutive light-spot sizes at each mirror.

The software might be executed from either *.exe or *.py file. The second option requires Python 3 with such basic modules as matplotlib, numpy and datetime (see Appendix 2 for more details).

The file named mps.txt contains the basic information required to start the simulation. Its structure is shown in Subsection 4.1 below. The information is divided into two parts. The first one, named as FIXED PARAMETERS, describes the data which is impossible to adjust during the experiment (e.g., the mirror diameters, the radii of mirror curvatures, the holes radii and their distances from the mirror centres). Moreover, the constant values of the input beam radius and the beam spread angle at the input hole should be declared in this file. The second part of the mps.txt file, which begins from the line ADJUSTABLE PARAMETERS, contains the data easy changeable by the sliders on screen (e.g., the laser-beam injection angles, the mirror separation, the mirror tilts and the rotation angles).

After launching the program, a graphic interface appears (see Fig. 4). The light spot distributions on the internal side of each mirror (M1 and M2) are shown on the top of the window. The hole positions are also marked here.

One assumes that the laser beam enters the cell through the hole in the M1 mirror. Depending on the adjustment and the type of the M2 mirror (entire or not), this hole can also play a role of an output aperture (see Fig. 1a). However, the modelling for the configuration presented in Fig. 1b is also possible (see Fig. 4 and Fig. 5). A display for numeration of laser spots can be switched by clicking at Num. On (or Num. Off) button in the top-left part of the interface. This makes the spot distribution more perspicuous.

The simulation can be done in the two modes. A Ray is line option ignores the thickness and the spread of the laser beam, while a Ray is a beam mode takes them into account. Selecting this option unlocks the two bottom sliders. A Reset button restores the adjustable parameters to the initial values determined in mps.txt file.

Green sliders in the bottom part of the picture can be used to change the adjustable parameters. Their actual values are shown on the right side of the sliders. The output data is located under Reset and Save buttons. They contain the simulation results, i.e. Optical path, Number of passes and the angles of the light beam leaving the cavity. In addition, information is displayed on whether the laser beam comes out cleanly through the hole $(h O K)$ or hits its edge ( $h E d g e$ ). Input and output data can be saved to text file by pressing the Save button. 


\section{Illustrative example}

We describe a typical usage of our MUPASS_GUI_simulation to demonstrate the main features of the software and illustrate the process of design of a multi-pass cavity. The procedure consists of the three steps: (i) modification of mps.txt file, (ii) launching of the main program and optimization of the parameters, and (iii) saving data. MUPASS GUI has been tested with the following versions of modules: python 3.7.6, numpy 1.18.1 and matplotlib 3.1.3. Reading a README.txt file before proceeding and executing the program is strongly recommended.
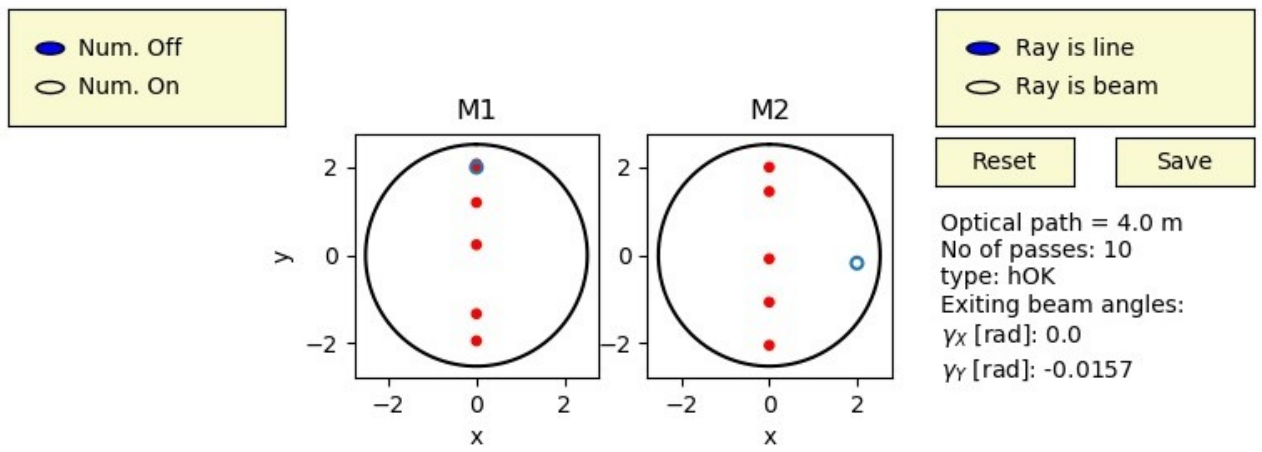

Optical path $=4.0 \mathrm{~m}$

No of passes: 10

type: hOK

Exiting beam angles:

$\gamma_{x}[\mathrm{rad}]: 0.0$

$\gamma_{Y}[\mathrm{rad}]:-0.0157$

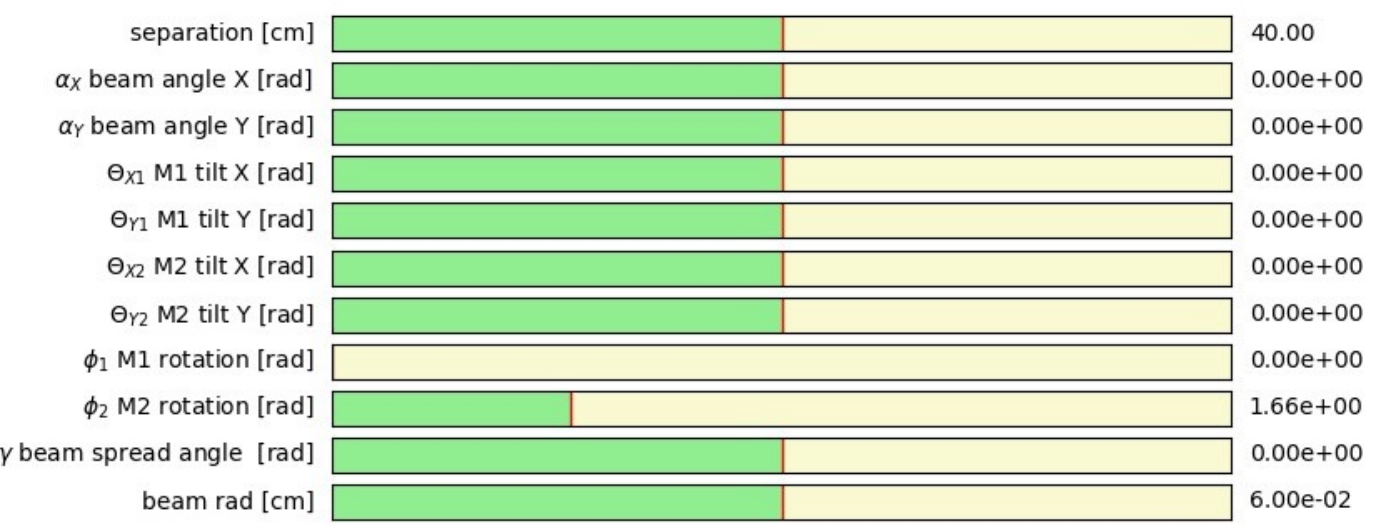

Fig. 4. Illustration of a main window of our program ${ }^{1}$.

\subsection{Modifying mps.txt file}

The mps.txt file is used to describe the fixed parameters and their ranges. These parameters must be established before the main program is launched. The following important lines are written in the mps.txt file:

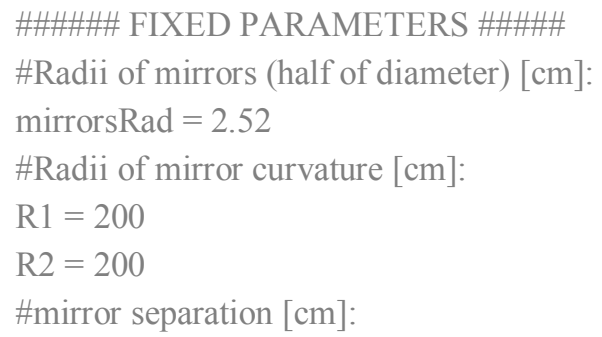

${ }^{1}$ Magnifier and home buttons from the Windows bottom bar (not shown in Fig. 4 and Fig. 5) can be used to adjust the view of mirrors. 


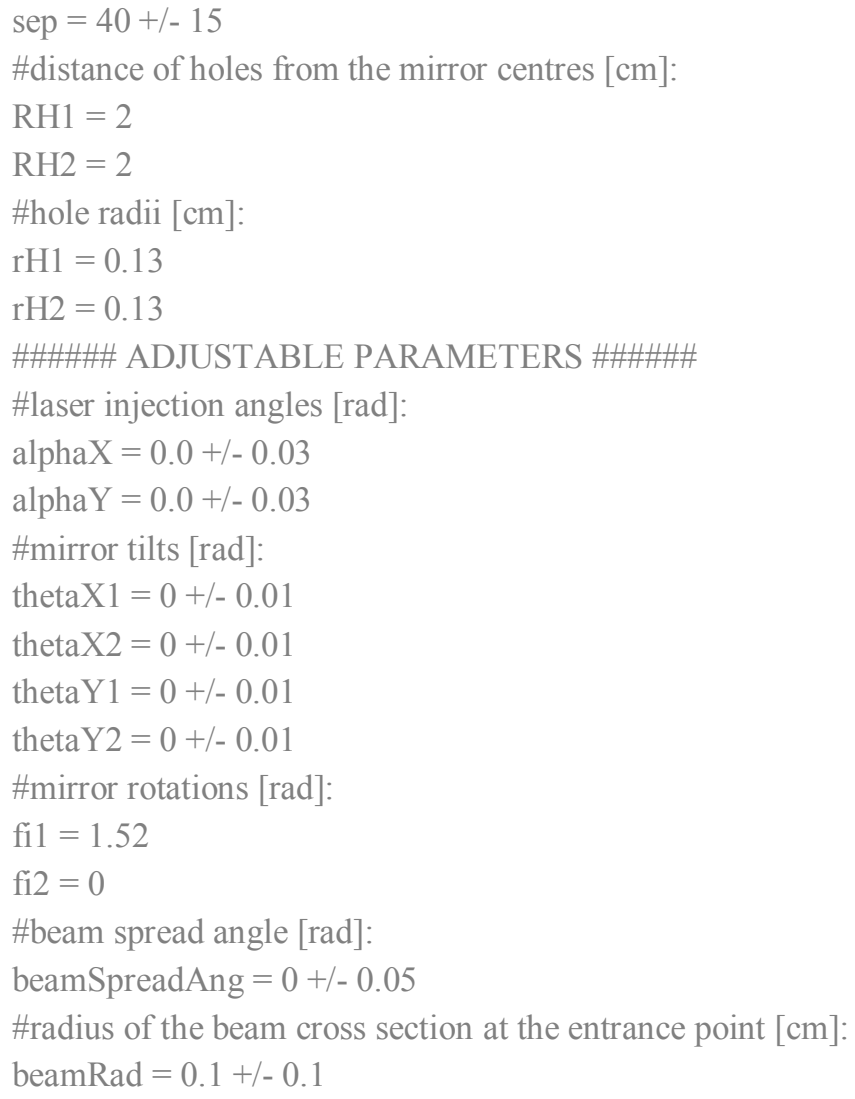

One must make sure that a previous content of the file has been removed. Then the mps.txt file has to be saved in the directory of main simulations. Note that this file is delivered in the software package. Finally, the library raytrace.py must be located in the same folder.

\subsection{Functions of the main program}

The main window of the program is displayed in Fig. 4. Adjusting the parameters is possible via shifting the sliders. Their actual values are displayed on the right side of the sliders. The spots on the mirrors move during this process and the resulting values are actualized in the section below the Save and Reset buttons. Numeration of the spots on the mirrors can be switched on or off by the Num. On/Off button. Note that the two parameters, beamSpreadRad and beamSpreadAng, are fixed in the mode conventionally denoted as Ray is line. In order to modify them, one has to change the simulation mode to what is conventionally referred to as Ray is beam.

Putting the parameters given in Fig. 5 on the screen activates elliptical patterns of laser spots on the mirrors. The actual values of the parameters are displayed in the main window. Such useful output parameters as Optical path length, No of Passes and Exit beam angles (i.e., the the $\gamma_{x}$ and $\gamma_{y}$ ) are shown below the Reset and Save buttons. On pressing the Save button one can save these parameters in the text file, which is named by date and hour. The other data collected from the screen, together with the fixed parameters, are also saved in this file, as illustrated below:

\#This is multipass saved file.

\#The following parameters where used: 


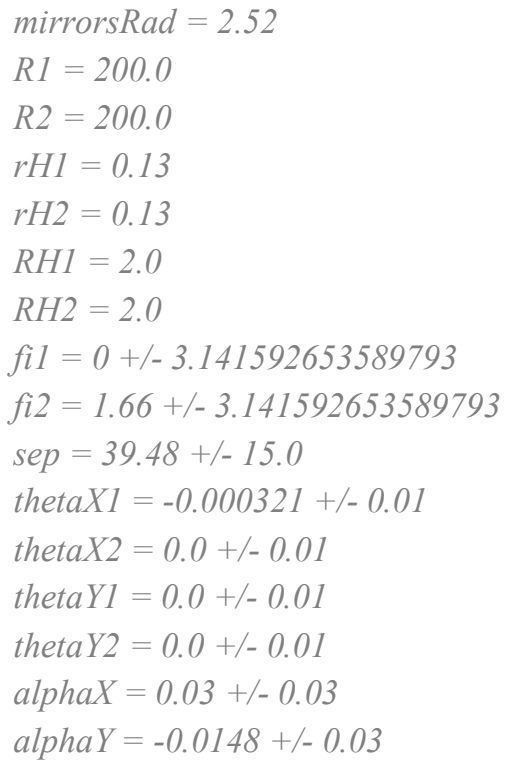

The output file is located in the main directory. The additional parameter displayed below the Reset and Save buttons, type: $h O K$ or $h E D G E$ or $O U T$, provides the information about the beam pass through the output hole in the mirror: its values are good, edge hitting or out of mirror, respectively.

Num. Off O Num. On

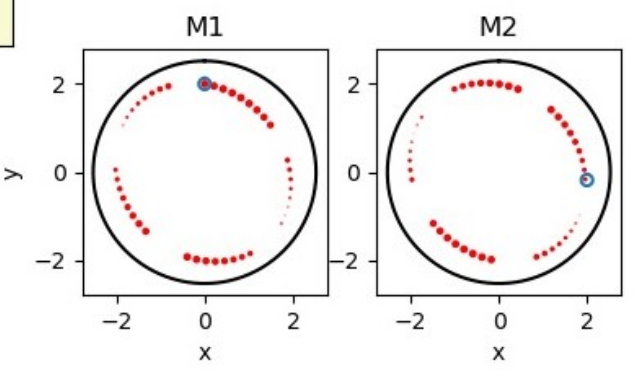

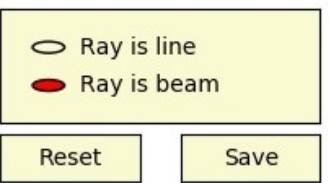

Optical path $=31.98 \mathrm{~m}$ No of passes: 81 type: hOK Exiting beam angles: $\gamma_{x}[\mathrm{rad}]: 0.0119$ $\gamma_{Y}[\mathrm{rad}]:-0.0312$

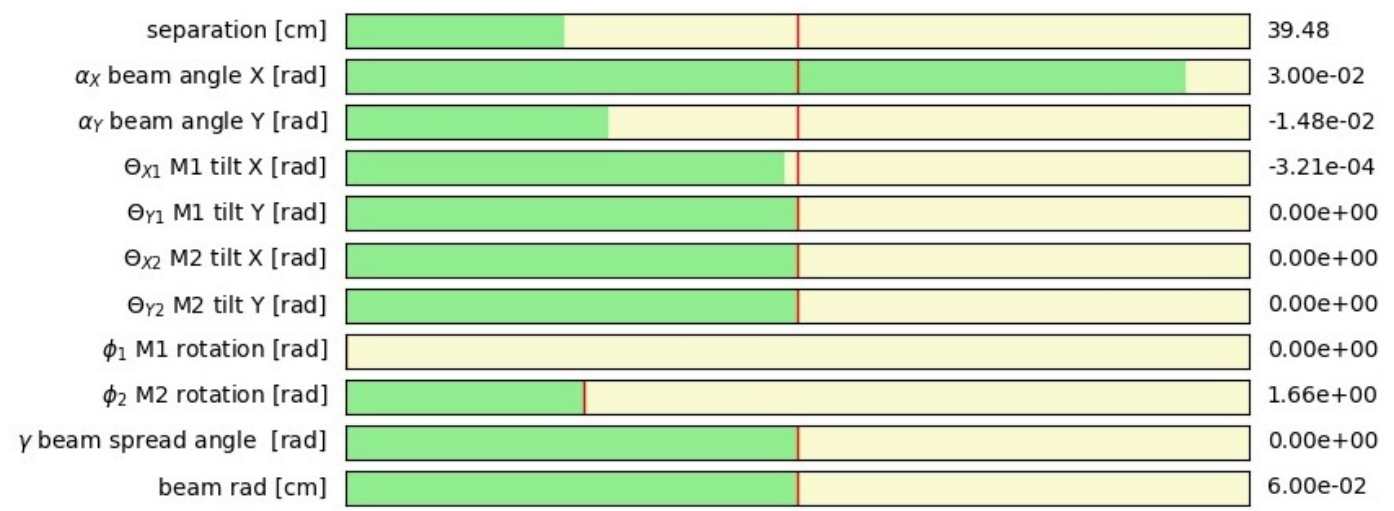

Fig. 5. Results of multi-pass cell optimization.

Ukr. J. Phys. Opt. 2021, Volume 22, Issue 1 


\section{Discussion}

Any comparison of our program with the software available in the literature is not a simple task, since we have found no specialized freeware program for designing and simulating multi-pass cells. There are many versatile environments aiding design of optical systems, although their application is rather problematic in our specific case. Zemax, FRED and OSLO are ready examples of such software. Unfortunately, they are chargeable programs. Moreover, their preparation for the multi-pass cell design and output-data presentation is laborious and timeconsuming [13].

On the other hand, as we have already noticed, increasingly better multi-pass cells are described in the literature, although no detailed data on their construction is provided, except for the decisive final parameters such as the effective path length [12, 14]. Any practical attempts to reproduce these cells are complex and might be affected by errors, often leading to necessity of serious construction corrections. This concerns not only optimization of the optical path length but also solving the problem of optical noise [15-18]. In this relation, our specified software under free-of-charge GPL license can simplify significantly the optical design and reduces the costs of the appropriate experiments. Moreover, our results can reduce the time needed to achieve the desired multi-pass optical equipment.

\section{Conclusion}

In the present work, we describe the interactive approach for designing the multi-pass optical cells. It provides the quantitative analysis of the multi-pass cells based upon the spherical concave mirrors. Our approach enables a researcher to find easily the optimal parameters for both the optical cavity and the optical system which forms the laser beam. The method and the work of the appropriate software have been tested for the experimental system that involves the 2 " mirrors with the radii of curvature equal to 40 and $200 \mathrm{~cm}$.

\section{Acknowledgement}

This work was supported by The Polish National Science Centre under research project No. 2016/23/B/ST7/03441.

\section{References}

1. Lizhu Zhang, Guang Tian, Jingsong Li and Benli Yu. 2014. Applications of absorption spectroscopy using quantum cascade lasers. Appl. Spectr. 68: 1095-1107.

2. Zahniser M S, Nelson D D, McManus J B, Herndon S C, Wood E C, Shorter J H, Lee B H, Santoni G W, Jiménez R, Daube B C, Sunyoung Park, Kort E A and Wofsy S C, 2009. Infrared QC laser applications to field measurements of atmospheric trace gas sources and sinks in environmental research: enhanced capabilities using continuous wave QCLs. Quantum Sensing and Nanophotonic Devices VI. Proc. SPIE. 7222: 1-9.

3. Kosterev A A, Mosely T S and Tittel F K, 2006. Impact of humidity on quartz-enhanced photoacoustic spectroscopy based detection of HCN. Appl. Phys. B. 85: 295-300.

4. Elia A, Lugarà P M, Di Franco $\mathrm{C}$ and Spagnolo V, 2009. Photoacoustic techniques for trace gas sensing based on semiconductor laser sources. Sensors. 9: 9616-9628.

5. Wang Chuji and Peeyush Sahay, 2009. Breath analysis using laser spectroscopic techniques: breath biomarkers, spectral fingerprints, and detection limits. Sensors. 9: 8230-8262.

6. Harren F J M, Berkelmans R, Kuiper K, te Lintel Hekkert S, Scheepers P, Dekhuijzen R, Hollander P and Parker D H, 1999. On-line laser photoacoustic detection of ethene in exhaled 
air as biomarker of ultraviolet radiation damage of the human skin. Appl. Phys. Lett. 74: 1761-1763.

7. Demtröder Wolfgang. Laser spectroscopy. Berlin Heidelberg: Springer-Verlag (1973).

8. White J U, 1942. Long optical paths of large aperture. J. Opt. Soc. Amer. 32: 285-288.

9. Herriott D, Kogelnik H and Kompfner R. 1964. Off-axis paths in spherical mirror interferometers. Appl. Opt. 3: 523-526.

10. Altmann J, Baumgart R and Weitkamp C, 1981. Two-mirror multipass absorption cell. Appl. Opt. 20: 995-999.

11. Herriott D R and Schulte H J, 1965. Folded optical delay lines. Appl. Opt. 4: 883-889.

12. Kun Liu, Lei Wang, Tu Tan, GuishiWang, Weijun Zhang, Weidong Chen and Xiaoming Gao, 2015. Highly sensitive detection of methane by near-infrared laser absorption spectroscopy using a compact dense-pattern multipass cell. Sens. Actuat. B: Chemical. 220: 1000-1005.

13. Tuzson B, Mangold M, Looser H, Manninen A and Emmenegger L, 2013. Compact multipass optical cell for laser spectroscopy. Opt. Lett. 38: 257-259.

14. Krzempek K, Jahjah M, Lewicki R, Stefański P, So S, Thomazy D and Tittel F K, 2013. CW DFB RT diode laser-based sensor for trace-gas detection of ethane using a novel compact multipass gas absorption cell. Appl. Phys. B. 112: 461-465.

15. Silver J A and Stanton A C, 1988. Optical interference fringe reduction in laser absorption experiments. Appl. Opt. 27: 1914-1916.

16. McManus B J and Kebabian P L, 1990. Narrow optical interference fringes for certain setup conditions in multipass absorption cells of the Herriott type. Appl. Opt. 29: 898-900.

17. Mangold M, Tuzson B and Emmenegger L, 2017. Method for reducing interference fringes in laser spectroscopy measurements using an absorption mask in combination with multi-pass optical cells. U.S. Patent No. 9,638,624.

18. Lim Lee, Kwang-Hoon Ko, Taek-Soo Kim and Do-Young Jeong, 2010. Reduction of fringe noise in a multi-pass absorption cell by using the wavelength modulation technique. J. Kor. Phys. Soc. 57: 364-368.

A. Belina Brzozowski, M. Winkowski, T. Stacewicz. 2021. Software for design and analysis of multi-pass absorption cells. Ukr.J.Phys.Opt. 22: 1 - 11. doi: 10.3116/16091833/22/1/1/2021

Анотація. Представлено інтерактивний розрахунковий підхід та відповідне програмне забезпечення для проектування багатопрохідних оптичних комірок. Наш метод виконує аналіз таких комірок для будь-яких наборів увігнутих сферичних дзеркал і дає змогу знайти оптимальні параметри і лазерного резонатора, і оптичної системи, яка формує лазерний промінь.

Ukr. J. Phys. Opt. 2021, Volume 22, Issue 1 


\section{Appendix 1. Program algorithm}

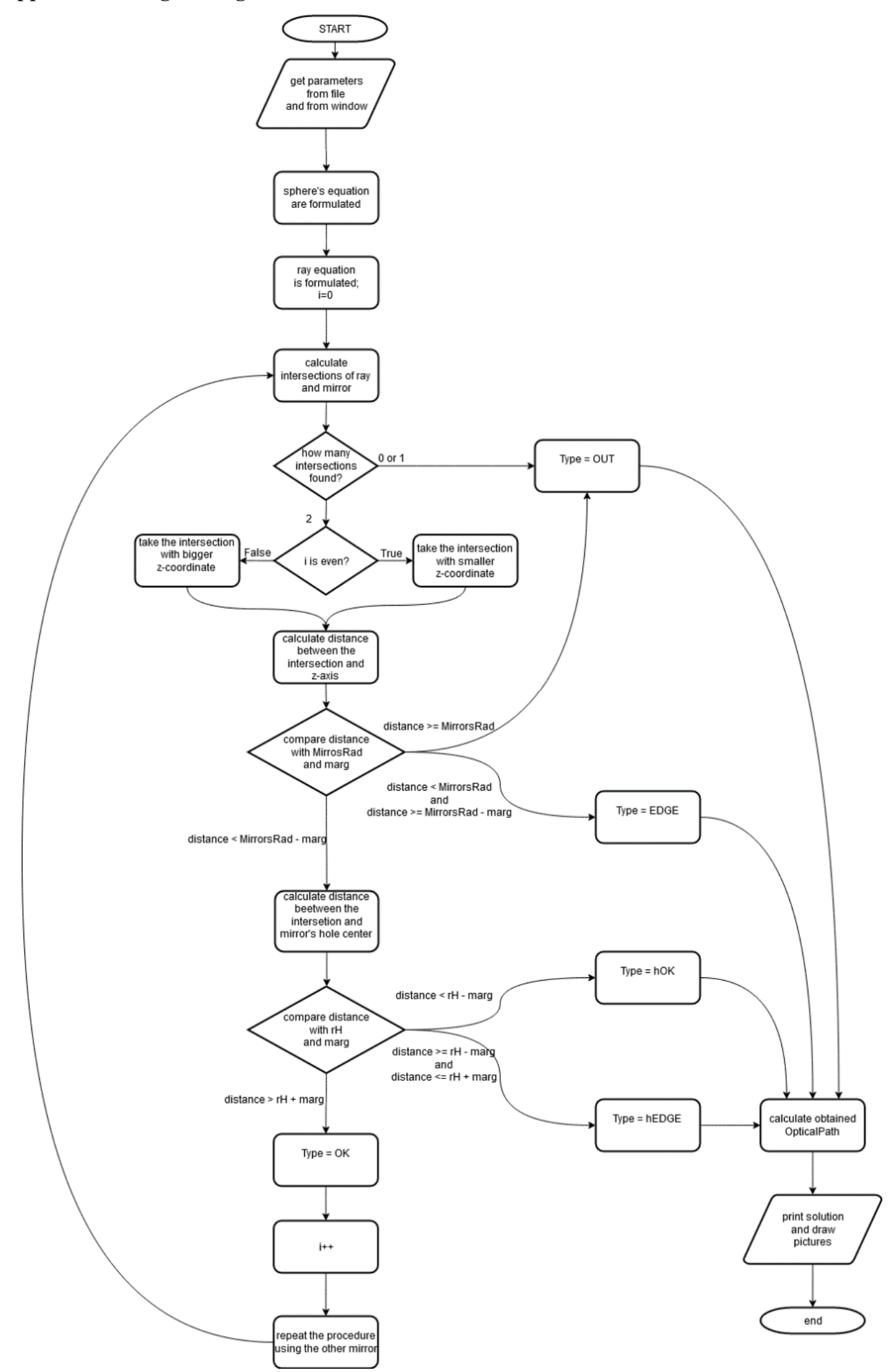


Appendix 2. Some software specifications

\begin{tabular}{|l|l|}
\hline Current software version & 1.0 \\
\hline $\begin{array}{l}\text { Permanent link to executables of this } \\
\text { version }\end{array}$ & $\begin{array}{l}\text { https://github.com/antonio- } \\
\text { panaro/MUPASS_GUI_simulation.git }\end{array}$ \\
\hline Legal software license & GNU GPL \\
\hline Computing platforms / Operating Systems & PC \\
\hline Installation requirements and dependencies & Python3 with NumPy and MatPlotLib \\
\hline Support email for questions & a.belina-brzoz@student.uw.edu.pl \\
\hline
\end{tabular}

